\title{
A Diffuse Interface Model of Interfaces: Grain Boundaries in Silicon Nitride
}

\author{
Catherine M. Bishop ${ }^{\mathrm{a}, \mathrm{b}, *}$ Rowland M. Cannon ${ }^{\mathrm{c}}$ \\ W. Craig Carter ${ }^{b}$

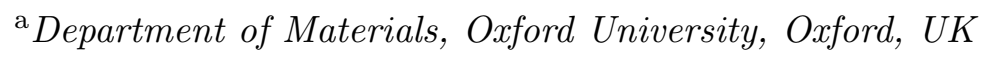 \\ ${ }^{\mathrm{b}}$ Department of Materials Science and Engineering, Massachusetts Institute of \\ Technology, Cambridge, MA, USA \\ ${ }^{\mathrm{c}}$ Materials Sciences Division, Lawrence Berkeley National Laboratory, Berkeley, \\ $C A, U S A$
}

\begin{abstract}
A diffuse-interface model for interfaces in multi-component systems with energetic contributions from chemistry, defects, structure, orientation, electrostatics and gradients is proposed. The energy minimizing profiles of planar grain boundaries in the pseudo-binary $\mathrm{SiO}_{2}-\mathrm{SiN}_{4 / 3}$ system are calculated in the $\mathrm{SiN}_{4 / 3}$-rich single-phase field. Intergranular films are found to be stable below the eutectic temperature. Evidence of first-order grain boundary order-disorder transitions is found in misorientation and chemical potential space. Interface transitions predicted with the model can be plotted on equilibrium phase diagrams to produce "interfacial phase diagrams." These could be a tool for designing processing routes to optimize bulk, polycrystalline material properties through control of grain boundary characteristics.
\end{abstract}

Key words: grain boundary wetting, thermodynamics, interface transitions, grain 


\section{Introduction}

Independent experimental observations establish that silicon nitride grain boundaries are typically glassy intergranular films (IGFs) containing $\mathrm{Si}, \mathrm{N}, \mathrm{O}$, and any dopants [1-9]. The IGFs have equilibrium thicknesses of 1-2nm, have thicknesses that vary with chemical potential of the additives but that are independent of the amount of glassy material in the sample, and have compositions that differ from those observed at glassy pockets at grain boundary junctions and from those predicted by equilibrium phase diagrams. Furthermore, IGF atomic structures deduced from microscopy observations suggest that, while the lack of long-range atomic structure identifies them as amorphous, short-range order is induced and influenced by the abutting crystalline grains $[10,11]$.

While IGFs occupy a relatively small volume fraction in a silicon nitride material, IGFs have a large-and sometimes dominant-influence on mechanical, creep, and transport properties [12-17]. Because similar IGFs are detected in other ceramic systems, such critical dependence of properties on IGFs should be expected and is indeed observed [18-28].

\footnotetext{
* Department of Materials, Oxford University, Parks Road, Oxford, OX1 3PH, UK. Phone $+44(0) 1865273672$ Email address: catherine.bishop@materials.ox.ac.uk (Catherine M. Bishop).
} 
Clarke developed a model that accounts for equilibrium IGF thicknesses [29]. His model describes the stability as a force balance between an applied (or capillary) pressure and the total pressure deriving from steric, adsorption, hydrogen bonding, electrical double layer, and dispersion contributions. Subsequently, Clarke included specific models for steric pressures in a diffuse interface theory [29], double layer pressures using DLVO theory [30], and osmotic forces from composition gradients [31]; these act as disjoining forces in these formulations. A key recognition was that the London dispersion forces could act to prevent complete wetting of the films and instead formation of equilibrium films as the grain boundary. Each model predicts stable film thicknesses of 1-2nm for plausible physical parameters. Subsequent models and numerical investigations amplified the importance of the steric term and the chemical effect of glass modifiers on steric behavior [32-34]. However, these models have largely ignored describing the actual IGF compositions, and especially have ignored the temperature dependences which should be interesting. It may be expected that there is only a range of temperatures in which the IGFs are stable.

A general model for IGFs could be used to formulate processing conditions for IGF characteristics in order to optimize material properties. However, a modelling framework that couples all of the relevant physical phenomena outlined by Clarke is not currently available. The diversity of physical influences on the stability and characteristics of IGFs underscores the difficulty of formulating such a predictive model which must also include the thermodynamics of the constituent materials. Direct atomistic calculations produce useful insights about stable structures or configurations [35-38], but only for small parts of specific systems using postulated interatomic potentials. In addition, these 
atomistic computations cannot yet predict the general trends in dependence of the IGF structures and compositions on temperature or additive chemical potential

In this paper, we present a diffuse interface model that accounts for chemistry, structure, defects, and electrostatic forces present at materials interfaces. The empirical parameters in our model must be tuned for particular systems with experimental data. Our continuum formulation permits straightforward generalization and allows the simulation of large systems from bulk data. The model is demonstrated for grain boundaries in silicon nitride ceramics with a minimal number of parameters.

\section{The Diffuse-Interface Model for Grain Boundaries in Multicom- ponent Systems}

The total free energy can be obtained by the spatial integration of a free energy density that is a function of a set of specified fields and their gradients. That free energy density is composed of two parts: the first is the free energy of a homogeneous system, and the second is the lowest-order inhomogeneous contribution to the energy density. In our model the homogeneous free energy density is a function of composition, structure, and defect density. The inhomogeneous contribution depends on gradients in the composition, structure, and electrostatic potential $[39,40]$. Finally because our materials are crystalline we include gradients in crystallographic orientation [41].

The homogeneous free energy density binds the model to the thermodynamics that predict an equilibrium phase diagram. Structural, compositional, orien- 
tational, and electrostatic fields bind the model to the microscopic physics associated with the grain boundary.

\subsection{Free Energy Functional}

We consider an isothermal, constant volume system with $N$ neutral species and $M$ (probably charged) defect species. There is a uniform site density and a fixed number of sites. Suppose that the site fractions $X_{i}(i=1, \ldots, N)$ for the neutral species and $X_{j}^{d}(j=1, \ldots, M)$ for the defect species are spatially variable fields subject to $\sum_{i}^{N} X_{i}(\vec{x})+\sum_{j}^{M} X_{j}^{d}(\vec{x})=1,0 \leq X_{i}(\vec{x}) \leq 1$, and $0 \leq X_{j}^{d}(\vec{x}) \leq 1$ at each position $\vec{x}$. As will be described below, we use a thermodynamic model based largely on neutral associated complexes, and then focus directly on the electrostatic consequences of the presence of sets of charged species, either conceived as solutes or defects, which will comprise a small minority of the total. Some approximations regarding the latter will be made in order to allow a treatment that smoothly covers both the solid and disordered material, which will be the subject of a later paper.

We suppose that the composition fields are continuous and therefore a coarsegraining is implicit in their definition. As only defects contribute electrostatic charge, then the charge density field, $\rho(\vec{x})$, is a function of defect composition $\rho\left(X_{j}^{d}\right)=\frac{\mathcal{F}}{V_{m}} \sum_{j}^{M} Z_{j} X_{j}^{d}$ where $\mathcal{F}$ is Faraday's constant, $Z_{j}^{d} e$ is the charge of the

$j^{\text {th }}$ defect with $e$ the magnitude of the electron charge, and $V_{m}$ is the molar site volume. Local charge density contributes to energy density with $\rho(\vec{x}) \phi(\vec{x})$ where $\phi(\vec{x})$ is the local electrostatic potential. If $\phi(\vec{x})$ is spatially variable, there is an additional contribution to energy density through polarization. 
We suppose that the material can adopt either a crystalline or amorphous structure and we use a structural field that describes the degree of crystallinity in this range, $0 \leq \eta(\vec{x}) \leq 1$. For example, a low angle grain boundary consisting of a set of dislocations would have a coarse-grained core value of $1>\eta \neq 0$ or a liquid epitaxially solidifying on a crystalline phase would have a boundary layer of decreasing $\eta$. In any region where $\eta(\vec{x})>0$, the crystallographic orientation (with respect to a reference crystal) can be described by three angles, which we call $\vec{\theta}(\vec{x})$. In the absence of applied fields, the homogeneous free energy cannot depend on the value of $\vec{\theta}$, but if $\vec{\theta}(\vec{x})$ is spatially variable, as in the case of a grain boundary, then the inhomogeneous part of the free energy density will depend on gradients of $\vec{\theta}(\vec{x})$. These fields were introduced elsewhere [41] and methods for their analytic [42,43] and experimental $[44,45]$ coarse-graining have been suggested.

The total energy of the system is

$$
\begin{aligned}
F\left[X_{i}, X_{j}^{d}, \phi, \eta, \theta ; T, V\right]= & \int_{V}\left[f^{\text {hom }}\left(X_{i}, X_{j}^{d}, \phi, \eta ; T\right)\right. \\
& \left.+f^{\text {inhom }}\left(X_{i}, X_{j}^{d}, \eta, \vec{\theta}, \nabla X_{i}, \nabla X_{j}^{d}, \nabla \phi, \nabla \eta, \nabla \vec{\theta} ; T\right)\right] d \vec{x}
\end{aligned}
$$

where the three-dimensional form of integration expresses the generality of the method; however, in this paper, only one-dimensional calculations are presented. To simplify discussion, we treat planar boundaries and suppose that the misorientation across the boundary can be described by a single angle, $\theta(\vec{x})$, as would be the case for a tilt or twist boundary. The temperature is $T$ and the total volume of the system is $V$. We assume a uniform, isotropic, and linearly polarizable material with dielectric constant $\epsilon[39,40]$. 
Therefore, in this model we take

$$
f^{\text {hom }}=f\left(X_{i}, X_{j}^{d}, \eta ; T\right)+\rho\left(X_{j}^{d} ; Z_{j}^{d}\right) \phi
$$

where $f\left(X_{i}, X_{j}^{d}, \eta ; T\right)$ is the chemical contribution to the Helmholtz free energy density and

$$
\begin{gathered}
f^{\text {inhom }}=-\frac{1}{2} \epsilon(\nabla \phi)^{2}+\frac{1}{2} \sum_{i=1}^{N} \kappa_{\mathrm{i}}^{2}\left(\nabla X_{i}\right)^{2}+\frac{1}{2} \sum_{j=1}^{M} \kappa_{\mathrm{j}}^{2}\left(\nabla X_{j}^{d}\right)^{2} \\
+s g(\eta)|\nabla \theta|+\frac{1}{2} \beta^{2} h(\eta)(\nabla \theta)^{2}+\frac{1}{2} \nu^{2}(\nabla \eta)^{2} . \\
\text { with } g(\eta)=\frac{1}{2} \eta^{2} \text { and } h(\eta)=1
\end{gathered}
$$

The functional expansion coefficients (or gradient coefficients) $\kappa_{\mathrm{i}}^{2}, \kappa_{\mathrm{j}}^{2}, s g(\eta)$, $\beta^{2} h(\eta)$, and $\nu^{2}$ are generally functions of all the field variables and their gradients; however any anisotropic directional dependence implied by dependence on gradient direction is inconsequential in a one-dimensional calculation. The forms of electrostatic potential gradient $[39,40]$ and structural and orientation gradient [41] terms are justified elsewhere.

Equilibrium phase diagrams are produced by common tangent constructions on $f\left(X_{i}, X_{j}^{d}, \eta ; T\right)$ that provide the connection to bulk thermodynamics for a particular material system. Equilibrium defect populations are determined by thermodynamics as well, but their energies are not usually written explicitly. Because their populations could be significant at interfaces, they are included explicitly in $f\left(X_{i}, X_{j}^{d}, \eta ; T\right)$. Previous analyzes have shown how spatial variations in charge density can be stabilized if coupled to chemical and mechanical contributions to free energy $[46,47,39]$.

As is common for ionic compounds, equivalence salt diagrams could be used to reduce the number of independent components if charge density were fixed [48]. 
However, local charge density variations necessitate that free energy density expressions must be extended to charged combinations of ionic species and simultaneously reproduce known phase diagrams for homogeneous phases. It is convenient to employ the associate model from the CALPHAD literature [49] with stable, neutral compounds for the $X_{i}(\vec{x})$. Therefore, non-zero $X_{j}^{d}(\vec{x})$ provide the extension to non-zero charge. Each of the $N-1$ independent $X_{i}$ has a chemical potential, $\mu_{i}$, that can be calculated by the intercept of the tangent plane to the equilibrium free energy surface with the $X_{i}=1$ line. The defect species have electrochemical potentials, $\mu_{j}^{d}$, where the chemical contribution is calculated as for $\mu_{i}$. By specifying the full set of $\mu_{i}$ and $\mu_{j}^{d}$, it is possible to fix the state of a homogeneous open system in equilibrium with a reservoir of species with these potentials. This scheme is similar to the compound energy formalism [50]. The defect species have enthalpic and entropic contributions to the homogeneous free energy and their populations will be very small at bulk homogeneous equilibrium. At interfaces these defect concentrations can contribute to locally charged regions, and double layer formation is expected.

\subsection{Dimensionless Form and Equilibrium Equations}

The total free energy can be normalized as follows

$$
\begin{aligned}
\hat{F}\left[X_{i}, X_{j}^{d}, \hat{\phi}, \eta, \theta ; T, \hat{V}\right] \equiv & \frac{V_{m}}{R T L^{3}} F\left[X_{i}, X_{j}^{d}, \phi, \eta, \theta ; T, V\right] \\
= & \int_{\hat{V}}\left\{\hat{f}\left(X_{i}, X_{j}^{d}, \eta ; T\right)+\hat{\rho} \hat{\phi}-\frac{1}{2} \hat{\epsilon}(\hat{\nabla} \hat{\phi})^{2}\right. \\
& +\frac{1}{2} \sum_{i=1}^{N} \hat{\kappa}_{\mathrm{i}}^{2}\left(\hat{\nabla} X_{i}\right)^{2}+\frac{1}{2} \sum_{i=j}^{M} \hat{\kappa}_{\mathrm{j}}^{2}\left(\hat{\nabla} X_{j}^{d}\right)^{2} \\
& \left.+\frac{1}{2} \hat{s} \eta^{2}|\hat{\nabla} \theta|+\frac{1}{2} \hat{\beta}^{2}(\hat{\nabla} \theta)^{2}+\frac{1}{2} \hat{\nu}^{2}(\hat{\nabla} \eta)^{2}\right\} d \hat{x}
\end{aligned}
$$


where the dimensionless variables are defined as

$$
\begin{array}{lll}
\hat{\phi} \equiv \phi \frac{\mathcal{F}}{R T} & \hat{\epsilon} \equiv \epsilon \frac{R T V_{m}}{\mathcal{F}^{2} L^{2}} & \hat{\rho}\left(X_{j}^{d} ; Z_{j}^{d}\right) \equiv \rho\left(X_{j}^{d} ; Z_{j}^{d}\right) \frac{V_{m}}{\mathcal{F}}=\sum_{j=1}^{M} X_{j}^{d} Z_{j}^{d} \\
\hat{\kappa}_{\mathrm{i}}^{2} \equiv \kappa_{\mathrm{i}}^{2} \frac{V_{m}}{R T L^{2}} & \hat{\kappa}_{\mathrm{j}}^{2} \equiv \kappa_{\mathrm{j}}^{2} \frac{V_{m}}{R T L^{2}} \quad \hat{s} \equiv s \frac{V_{m}}{R T L} \quad \hat{\beta}^{2} \equiv \beta^{2} \frac{V_{m}}{R T L^{2}} \quad \hat{\nu}^{2} \equiv \nu^{2} \frac{V_{m}}{R T L^{2}}
\end{array}
$$

with $R$ the gas constant and $L$ a characteristic length.

We consider an open system which is specified by fixed values of the $\mu_{i}$ and $\mu_{j}^{d}$. Equilibrium is determined by the minimum of

$$
\hat{F}\left[X_{i}, X_{j}^{d}, \hat{\phi}, \eta, \theta ; T, \hat{V}\right]-\sum_{i=1}^{N} \mu_{i} \int_{\hat{V}} X_{i} d \hat{x}-\sum_{j=1}^{M} \mu_{j}^{d} \int_{\hat{V}} X_{j}^{d} d \hat{x}
$$

The equilibrium conditions that must be satisfied at each position follow from variational calculus:

$$
\begin{aligned}
& \frac{\partial \hat{f}\left(X_{i}, X_{j}^{d}, \eta ; T\right)}{\partial X_{i}}-\hat{\kappa}_{\mathrm{i}}^{2} \hat{\nabla}^{2} X_{i}=\mu_{i} \\
& \frac{\partial \hat{f}\left(X_{i}, X_{j}^{d}, \eta ; T\right)}{\partial X_{j}^{d}}+Z_{j}^{d} \hat{\phi}-\hat{\kappa}_{\mathrm{j}}^{2} \hat{\nabla}^{2} X_{j}=\mu_{j}^{d} \\
& \frac{\partial \hat{f}\left(X_{i}, X_{j}^{d}, \eta ; T\right)}{\partial \eta}+\hat{s} \eta|\hat{\nabla} \theta|-\hat{\nu}^{2} \hat{\nabla}^{2} \eta=0 \\
& \hat{\nabla} \cdot\left[\frac{1}{2} \hat{s} \eta^{2} \frac{\hat{\nabla} \theta}{|\hat{\nabla} \theta|}\right]+\hat{\beta}^{2} \hat{\nabla}^{2} \theta=0 \\
& \hat{\rho}+\hat{\epsilon} \hat{\nabla} \hat{\phi}=0
\end{aligned}
$$

Equation 10 is Coulomb's Law from electrostatics as noted by Bishop et al [39]. 


\subsection{Homogeneous Energy Model}

A reasonable choice for the Helmholtz free energy density $f\left(X_{i}, X_{j}^{d}, \eta ; T\right)$ is a regular solution model

$$
\begin{aligned}
f\left(X_{i}, X_{j}^{d}, \eta ; T\right) & =\sum_{i=1}^{N} X_{i} \mu_{i}^{\circ}(\eta ; T)+\sum_{i=1}^{M} X_{j}^{d} \mu_{j}^{d, \circ}(\eta ; T) \\
& +\frac{R T}{V_{m}}\left(\sum_{i=1}^{N} X_{i} \ln X_{i}+\sum_{i=1}^{M} X_{j}^{d} \ln X_{j}^{d}\right) \\
& +\sum_{i=1}^{N} \sum_{k=i+1}^{N} \omega_{i k}(\eta ; T) X_{i} X_{k}+\sum_{j=1}^{M} \sum_{k=j+1}^{M} \omega_{j k}^{d} X_{j}^{d}(\eta ; T) X_{k}^{d} \\
& +\frac{1}{2} \sum_{i=1}^{N} \sum_{j=1}^{M} \omega_{i j}^{\times}(\eta ; T) X_{i} X_{j}^{d} .
\end{aligned}
$$

The first two sums contain reference chemical potentials and defect formation energies; the third and fourth terms are ideal entropies of mixing. The final terms are the regular solution energies of mixing, but are assumed to be functions of crystallinity and temperature.

The reference chemical potentials and defect formation energies, $\mu_{i}^{\circ}(\eta ; T)$ and $\mu_{j}^{d, \circ}(\eta ; T)$ respectively, are dependent on the local structure. Wheeler et al. [51] have introduced a convenient interpolation scheme, Eqs. 12 for the chemical potentials, for the free energy density between the solid and liquid functions, $f_{i}^{s}(T)$ and $f_{i}^{l}(T)$, which can be correlated with thermodynamic data [52].

$$
\begin{gathered}
\mu_{i}^{\circ}(\eta ; T)=f_{i}^{l}(T)+W_{i} q(\eta)+\left(f_{i}^{s}(T)-f_{i}^{l}(T)\right) p(\eta) \\
\text { where } q(\eta) \equiv \frac{1}{2} \eta^{2}(1-\eta)^{2} \\
\text { and } p(\eta) \equiv \eta^{3}\left(10-15 \eta+6 \eta^{2}\right)
\end{gathered}
$$

Local minima are assumed for the liquid, $\eta=0$, and perfectly crystalline, $\eta=1$, states and $W_{i}$ is a parameter that controls the height of the energy 
barrier between these local minima, Fig. 1. For pure associates, the free energy densities of the solid and liquid phases are $\mu_{i}^{\circ}(1 ; T)=f_{i}^{s}(T)$ and $\mu_{i}^{\circ}(0 ; T)=$ $f_{i}^{l}(T)$. These reference energies are tabulated in the literature. As shown below, the amorphous phase will be treated as the extrapolation of the liquid phase well below the melting temperature. The free energy of vitrification should be related to $W_{i}$. But we have not attempted to use physical values here, just as we have not yet fitted values for $\nu, \beta$, etc.

\subsection{Application to the Pseudo-Binary Si-N-O System}

To develop a specific model for grain boundaries in silicon nitride containing small amounts of silica, we treat the binary alloy as a reduction of the Si-NO system. Simulations of this model demonstrate IGF behavior that can be compared to experimental observations. The distribution of charged defects is neglected below and will be treated in a subsequent paper.

With 3 ionic components, $\mathrm{Si}^{+4}, \mathrm{~N}^{-3}$ and $\mathrm{O}^{-2}$, the condition of charge neutrality implies that two are independent, but as these are expressed as site fractions, there is only one independent measure of composition. We choose the associates to be $\mathrm{SiN}_{4 / 3}$ and $\mathrm{SiO}_{2}$ and adopt the shorthand $\mathrm{SiN}_{4 / 3}=\mathrm{SN}$ and $\mathrm{SiO}_{2}=\mathrm{SO}$. The single independent composition field is $X_{S N}$ and $\hat{\kappa}^{2}=\hat{\kappa}_{\mathrm{SN}}^{2}+$ $\hat{\kappa}_{\text {SO }}^{2}$. The bulk free energy data are obtained from the literature: solid and liquid $\mathrm{SiO}_{2}$ and liquid $\mathrm{Si}_{3} \mathrm{~N}_{4}[53]$ and solid $\mathrm{Si}_{3} \mathrm{~N}_{4}$ [54]. The coefficient on the energy of mixing was obtained by $\mathrm{Gu}$ et al as $R T \omega_{S N, S O}(0 ; T)=45 \mathrm{~kJ} /$ mole [55]. The dimensionless energy barrier parameters are chosen to be $\hat{W}_{S N}=\hat{W}_{S O}=10$.

The normalized molar free energy density is 


$$
\begin{aligned}
\hat{f}\left(X_{S N}, \eta ; T\right)= & X_{S N}\left(\hat{\mu}_{S N}^{\circ}-\hat{\mu}_{S O}^{\circ}\right)+\hat{\mu}_{S O}^{\circ} \\
& +X_{S N} \ln X_{S N}+\left(1-X_{S N}\right) \ln \left(1-X_{S N}\right) \\
& +\hat{\omega}_{S N, S O}(\eta ; T) X_{S N}\left(1-X_{S N}\right)
\end{aligned}
$$

We assume the enthalpy of mixing to be lower in the liquid than the crystalline state with this functional form

$$
\hat{\omega}_{S N, S O}(\eta ; T)=\frac{1}{2} \hat{\omega}_{S N, S O}^{\circ}\left(1+\eta^{2}\right)
$$

This regular solution free energy results in the phase-diagram illustrated in Fig. 2. In this model, the stoichiometric line compound $\mathrm{Si}_{2} \mathrm{~N}_{2} \mathrm{O}$ is not present, as is typical of experimental observations in many systems [55]. Also we note that the above has made the solubilities in both solid phases small, and the results do not depend upon the actual level of solubility in the solids.

The form of the inhomogeneous energy is

$$
\hat{f}^{\text {inhom }}=\frac{1}{2} \hat{\kappa}^{2}\left(\hat{\nabla} X_{S N}\right)^{2}+\frac{1}{2} \hat{s} \eta^{2}|\hat{\nabla} \theta|+\frac{1}{2} \hat{\beta}^{2}(\hat{\nabla} \theta)^{2}+\frac{1}{2} \hat{\nu}^{2}(\hat{\nabla} \eta)^{2}
$$

which combined with Eqs. 13, 1and 5 gives the functional that is minimized at equilibrium. 


\subsection{Interface Boundary Conditions}

Equilibrium states are obtained by solutions to the PDEs Eqs. 6 - 10 and the boundary conditions which we choose to simulate a grain boundary

$$
\begin{array}{ccc}
\theta(\hat{x}=0)=0 & \frac{d \eta}{d \hat{x}}(\hat{x}=0)=0 & \frac{d X_{S N}}{d \hat{x}}(\hat{x}=0)=0 \\
\theta(\hat{x}=\infty)=\frac{\Delta \theta}{2} & \eta(\hat{x}=\infty)=1 & X_{S N}(\hat{x}=\infty)=X_{S N}^{e q}
\end{array}
$$

with the far-field compositions equal to the bulk equilibrium compositions, $X_{S N}^{e q}$, with corresponding specified chemical potentials, $\hat{\mu}_{S N}$ and $\hat{\mu}_{S O}$. The center of the boundary is located at $\hat{x}=0$ and we reduce the problem to the half-space. The infinite system was simulated by a finite system with width $\hat{W}$ where $\hat{W}$ was chosen by trial and error so that any variation in interfacial profile was sufficiently removed from $\hat{x}=\hat{W}$.

\subsection{Simulation Conditions}

The eutectic temperature in Fig. 2 is 1930K. One series of calculations was made above this eutectic temperature $(1950 \mathrm{~K})$ and another series performed below $(1900 \mathrm{~K})$. In each series, the misorientations $\Delta \theta$, specified by the boundary conditions, Eq. 16, were varied systematically and the equilibrium bulk compositions, specified by the chemical potentials, were varied in the singlephase phase-field up to the solubility limit. The misorientation is used as an input parameter because Kobayashi et al. showed that for ordered boundaries in single-component systems the interfacial energy is proportional to $\Delta \theta[41]$. This is true for the functional form of the gradient penalty that we use and so allows a demonstration of the trends with the grain boundary energy. More 
realistic functional dependences can be invoked for specific systems.

The length scale, $L=5 \cdot 10^{-11} \mathrm{~m}$, was used in the normalization given in Section 2.2. The gradient energy coefficients were given constant values so that at $T=1900 K$ the dimensionless gradient energy coefficients were $\hat{\kappa}^{2}=10$, $\hat{s}=20, \hat{\beta}^{2}=10$, and $\hat{\nu}^{2}=20$ and because the normalization is temperature dependent they are re-scaled for $1950 \mathrm{~K}$. These values for the gradient energy coefficients were chosen as they produce behavior similar to that observed in experiment. Methods for generating suitable gradient energy coefficients for particular material systems are discussed in the Section 4. The results from these calculations are presented in Section 3.

\subsection{Numerical Methods}

We find that simulated annealing methods [56] yield approximate solutions and converge in reasonable times. The simulated annealing was accomplished by approximating each field with a combination of cubic splines and tanh functions on a sub-interval of $0 \leq \hat{x} \leq \hat{W}$, Fig. 3. Each approximating function includes several independent parameters that are varied by Monte Carlo algorithms for the total energy as implemented in the simulated annealing technique. The use of tanh approximating functions permits the method to be compared to known double well diffuse interface profiles [57].

Each of the entire $X_{S N}$ and $\eta$ fields is approximated by two tanh functions (7 parameters) and cubic splines connected at $p$ nodes ( $p$ parameters). The $\theta$ field is approximated by a single-parameter tanh function with its center at $\hat{x}=0, \theta(\hat{x})=\frac{\Delta \theta}{2 \tanh (C \hat{W})} \tanh (C \hat{x})$. Thus, the solutions were obtained by 
minimizing the total energy, Eq. 5, with respect to the $2(7+p)+1$ degrees of freedom.

\section{Results}

The numerical results show two different classes of solutions. There are interfaces where the disordered region is narrow and the $\eta$ profile exhibits a cusp at the core. We will call this class the ordered interface solutions. In the second class of solutions the $\eta$ profile is flat in concentration and has a larger characteristic width. We will call these the disordered or IGF solutions. Examples of both classes appear in Fig. 4 .

The interfacial widths, $h$, are measured from the full-width half-maximum of the $\eta(x)$ profiles so that even the ordered interface solutions have a width of several $\AA$ with this definition. In addition to a measure of width, we characterize the adsorption of $\mathrm{SiO}_{2}, \Gamma$, by integrating the local $\mathrm{SiO}_{2}$ content minus its bulk equilibrium value. Finally, we report the interfacial energy, $\gamma$, which is the minimum value of Eq. 5 obtained by simulated annealing. This is the interfacial energy because of the subtraction of the bulk-reference energies. These results are presented in Figs. 5 and 6. The chemical potentials increase towards coexistence at $\hat{\mu}_{S O}^{c e}$.

The trends are the following: the $h$ are an increasing function of $\Delta \theta$ and $\hat{\mu}_{S O}$; $\gamma$ increases with $\Delta \theta$ and is a decreasing function of $\hat{\mu}_{S O}$; and $\Gamma$ is related to $h$ by the width of the miscibility gap for disordered films.

The ordered solutions are typically found at sub-eutectic temperatures, low $\hat{\mu}_{S O}$, or $\Delta \theta$. These solutions are characterized by low $\Gamma$ and a $\gamma$ which varies 
substantially with $\Delta \theta$ but not with $\hat{\mu}_{S O}$. The disordered solutions tend to occur at large $\Delta \theta$ and $\hat{\mu}_{S O} \rightarrow \hat{\mu}_{S O}^{c e}$. They are characterized by larger $\Gamma$ and $h$ and $\gamma$ that decrease rapidly with $\hat{\mu}_{S O}$.

In some cases, the simulated annealing algorithm results in two locally minimizing states for the same input parameters. These appear in Fig. 7(a) as multiple interfacial energies at the same value of chemical potential at a fixed misorientation. This effect is observed above and below the eutectic.

\section{Discussion}

The numerical calculations were performed on a grain boundary in a singlephase alloy of $\mathrm{Si}_{3} \mathrm{~N}_{4}$, Fig. 2. The thermodynamics of charged defects was included in the general theory, but neglected in the simulations. With bulk thermodynamic data for the silicon nitride-silica system, the simulations predict increased amounts of silica and reduced crystallinity at the grain boundary core. The gradient energy coefficients in Eq. 15 were chosen to correlate roughly with experimental observations but were not optimized. Nevertheless, we believe that these predictions of interfacial characteristics from a continuum thermodynamic model indicate that the diffuse-interface model provides

a useful means of generalizing and characterizing interfacial behavior [58]. Because the gradient energy coefficients were scaled to remove temperature dependence, we believe that the observed trends (misorientation dependent interface energy and interface width correlations with $\left.\hat{\mu}_{\mathrm{SO}}\right)$ are reliably predicted.

This method may be considered an extension of CALPHAD techniques to 
interfaces with a minimal number of additional empirically tuned parameters (e.g. gradient energy coefficients), which predicts the temperature and composition dependence of interfacial thickness and absorption. These predictions could be used as design parameters for polycrystalline materials for which grain boundaries control macroscopic material behavior.

The inclusion of thermodynamic interfacial characteristics onto phase diagrams was employed by Cahn in his analysis of surface absorption states in critical wetting [59]. Recently Tang et al [60,61], extended Cahn's critical wetting analysis to the diffuse interface functional similar to Eq. 4. Similar to the relatively thick and thin absorbed stable states predicted in the critical wetting theory, Tang predicts transitions between less and more disorder at grain boundaries. It was Tang's analysis that suggested the speculated spinodal curve in the $\gamma$ vs. $\hat{\mu}_{\text {SO }}$ plot in Fig. 7.

With regard to transitions that are observed in Fig. 7, our simulations fell into three categories. At low misorientations, only the ordered grain boundary states arise, with relatively low values of interfacial energy and thickness, independent of $\mu_{\mathrm{SO}}$. At high misorientations, there is a continuous transition of ordered to disordered grain boundary states, as suggested by the curve in Fig. 7(b). At intermediate misorientations, there are metastable grain boundary states and a first-order transition in grain boundary behavior as suggested by the curve in Fig. 7(a). These first-order transitions are accompanied by discontinuous jumps in thickness and adsorption level. This suggests that a nucleation barrier exists for the transition between the two grain boundary structures which could be used for empirical specification of $W_{i}$.

The results can be compared to observations in silicon nitride ceramics. Sta- 
ble, thick intergranular films are observed in many, but not all boundaries. Presumably, they are absent at boundaries of relatively low grain boundary energy. Experiments suggest that the films are not strictly amorphous, but some crystalline order is inherited from the abutting crystalline phases. These observations coincide with the disordered solutions in our model. However, the average composition of the disordered boundaries here is close to the eutectic liquid composition which contradicts experimental findings.

Models that derive from Clarke's force balance models [29-31] require an attractive force to stabilize an IGF. In this model, the increased homogeneous energy of the intergranular composition and structure when below the eutectic temperature contributes an attractive force. The intergranular layers would be completely wetting above the eutectic without at least a dispersion force being included. Experiments on silicon nitride grains dispersed in bulk glass [6] indicate that an attractive force persists above the eutectic. Our model for silicon nitride must be supplemented with other known attractive forces deriving perhaps from space charge [47] or coherency effects which could be introduced through the gradient energy coefficients.

All of the resultant field profiles from this work are symmetric about $x=0$ which restricts the above treatment to symmetric tilt boundaries. In order to generate non-symmetric relaxed fields, some symmetry relations must be introduced into the free energy statement. Rigorous methods for doing this can be related to the Cahn-Hoffman $\xi$-vector and have been derived for singlecomponent systems [62] and extended to multi-phase systems [63].

For very simple phase-field models, the gradient energy coefficients can be directly related to interfacial energy in the sharp interface limit [51]. For more 
complex models, numerical algorithms must be used to correlate parameters with observation. Methods for extracting continuum measures of structure and chemistry from atomistic calculations could be used as a supplement to tune model parameters $[42,43]$. A way of tuning parameters using coarse-grained data from HRTEM has been suggested by Koch [64,45] and is presently being integrated with analytic methods.

Despite the inadequacies discussed above, the method presented in this paper does shed light on observed phenomena which have been heretofore unexplained. In particular, Wang and Chiang observed stable amorphous phases, representative of eutectic liquid, at grain boundaries solely processed below the eutectic temperature in Bi-doped $\mathrm{ZnO}$ [22]. These arise naturally when grain boundaries are coupled to thermodynamics of bulk phases and our results are interpreted as the $\mathrm{SiO}_{2}-\mathrm{Si}_{3} \mathrm{~N}_{4}$ analogue to this phenomenon.

\section{Summary}

We propose a thermodynamic diffuse-interface model for interfaces with energetic contributions from chemistry, defects, structure, orientation, electrostatics, and gradients. There is a small number of empirical parameters and bulk-equilibrium thermodynamics is incorporated.

The $\mathrm{SiO}_{2}-\mathrm{SiN}_{4 / 3}$ pseudo-binary system is examined in the absence of defects and electrostatic contributions to the energy. In general, for low misorientations and chemical potentials far from coexistence, ordered boundaries are stable and for large misorientations and chemical potentials approaching coexistence, disordered boundaries are stable. For certain inputs, the simulated 
annealing technique uncovers two locally stable energy minima which imply a first-order order-disorder transition.

Stabilized, thick, liquid-like films at grain boundaries are found below the eutectic temperature in the analogue to experimental results on Bi-doped $\mathrm{ZnO}$ [22]. Our model is the first to examine an IGF-forming system with energetics that account for the coupling of chemistry, structure and misorientation. While the calculations in this paper are performed on a model that neglects defects and electrostatics, we expect the trends predicted here to persist when these energetic contributions are included in future work.

Above the eutectic at liquid-solid coexistence, our model gives complete wetting which disagrees with experiments [6]. Modification of the gradient energy coefficients and inclusion of electrostatic or London dispersion effects may correct this and delay the onset of complete wetting.

In single-phase polycrystalline alloys, grain boundaries are necessary defects that have an associated defect energy. Sharp and clean grain boundaries can activate additional degrees of freedom - in particular, absorption and structural transitions - when these decrease the interfacial energy. The efficacy of testing such observations and using them to generalize material phenomena is a benefit of the methods described in this paper. This approach could lead to the creation of interfacial phase diagrams which could be used to design processing routes to optimize the properties of real materials. 


\section{Acknowledgments}

CMB and WCC were supported by NSF Grant DMR-0010062 and the SingaporeMIT Alliance. RMC was supported by the Office of Basic Energy Science, Division of Materials Sciences and Engineering of Dept. of Energy under Contract No. DE-AC03-76F00098. The work described here was conducted in cooperation with the whole NANOAM collaboration whose European partners were funded from EU Commission Contract G5RD-CT-2001-00586. The discussions and hospitality of Dr. Dominique Chatain and of Profs. Adrian Sutton and David Cockayne are gratefully acknowledged by WCC and CMB.

\section{References}

[1] H. Gu, R. M. Cannon, M. Rühle, Composition and chemical width of ultrathin amorphous films at grain boundaries in silicon nitride, Journal of Materials Research 13 (1998) 376-387.

[2] H.-J. Kleebe, M. J. Hoffmann, M. Rühle, Influence of secondary phase chemistry on grain boundary film thickness in silicon nitride, Zeitschrift für Metallkunde 83 (1992) 610-617.

[3] I. Tanaka, H.-J. Kleebe, M. K. Cinibulk, J. Bruley, D. R. Clarke, M. Rühle, Calcium concentration dependence of the intergranular film thickness in silicon nitride, Journal of the American Ceramic Society 77 (1994) 911-914.

[4] I. Tanaka, J. Bruley, H. Gu, M. J. Hoffmann, H.-J. Kleebe, R. M. Cannon, D. R. Clarke, M. Rühle, Compositions and thicknesses of grain boundary films in cadoped silicon nitride ceramics, in: M. J. Hoffmann, G. Petzow (Eds.), Tailoring of Mechanical Properties of $\mathrm{Si}_{3} \mathrm{~N}_{4}$ Ceramics, Kluwer Academic Publishers, 
Amsterdam, 1994, pp. 275-289.

[5] H.-J. Kleebe, M. K. Cinibulk, I. Tanaka, J. Bruley, J. S. Vetrano, M. Rühle, High resolution electron microscopy studies on silicon nitride ceramics, in: M. J. Hoffmann, G. Petzow (Eds.), Tailoring of Mechanical Properties of $\mathrm{Si}_{3} \mathrm{~N}_{4}$ Ceramics, Kluwer Academic Publishers, Amsterdam, 1994, pp. 259-274.

[6] C.-M. Wang, X. Pan, M. J. Hoffmann, R. M. Cannon, M. Rühle, Grain boundary films in rare-earth-based silicon nitride, Journal of the American Ceramic Society 79 (1996) 788-792.

[7] H.-J. Kleebe, Structure and chemistry of interfaces in $\mathrm{Si}_{3} \mathrm{~N}_{4}$ materials studied by transmission electron microscopy, J. Ceram. Soc. Jpn 105 (1997) 453-475.

[8] R. M. Cannon, M. Rühle, M. J. Hoffmann, R. H. French, H. Gu, A. P. Tomsia, E. Saiz, Adsorption and wetting mechanisms at ceramic grain boundaries, Ceram. Trans. 118 (2000) 427-444.

[9] M. J. Hoffmann, H. Gu, R. M. Cannon, Influence of the interfacial properties on the microstructural development and properties of silicon nitride ceramics, in: Materials Research Society Proceedings, Vol. 586, Materials Research Society, Warrendale, PA, 2000, pp. 65-74.

[10] I. MacLaren, Improved visibility of thin amorphous intergranular films using Fourier filtering, in: S. McVitie, D. McComb (Eds.), Electron microscopy and microanalysis 2003, Institute of Physics, 2004, pp. 253-256.

[11] M. Döblinger, G. B. Winkelman, C. Dwyer, C. Marsh, A. I. Kirkland, D. J. H. Cockayne, M. J. Hoffmann, Structural and compositional comparison of $\mathrm{Si}_{3} \mathrm{~N}_{4}$ ceramics with different fracture modes, submitted to Acta Materialia (2005).

[12] G. Petzow, M. Herrmann, Silicon nitride ceramics, High Performance NonOxide Ceramics II Structure and Bonding 102 (2002) 47-167. 
[13] P. Sajgalik, J. Dusza, M. J. Hoffmann, Relationshop between microstructure, toughening mechanisms, and fracture toughness of reinforced silicon nitride ceramics, J. Am. Ceram. Soc. 78 (1995) 2619-2624.

[14] P. F. Becher, E. R. Fuller, P. Angelini, Matrix-grain-bridging contributions to the toughness of whisker-reinforced ceramics, J. Am. Ceram. Soc. 74 (1991) 2131-2135.

[15] H.-J. Kleebe, $\mathrm{SiC}$ and $\mathrm{Si}_{3} \mathrm{~N}_{4}$ materials with improved fracture resistance, Journal European Ceramic Society 10 (1992) 151-159.

[16] P. F. Becher, E. Y. Sun, K. P. Plucknett, K. B. Alexander, C.-H. Hsueh, H.-T. Lin, S. B. Waters, C. G. Westmoreland, E.-S. Kang, K. Hirao, M. E. Brito, Microstructural design of silicon nitride with imporved fracture toughness: I, effects of grain shape and size, J. Am. Ceram. Soc. 81 (1998) 2821-2830.

[17] R. L. Satet, M. J. Hoffmann, Impact of the intergranular film properties on microstructure and mechanical behavior of silicon nitride, Euro Ceramics VIII 264-268 (2004) 755-780.

[18] M. A. Gülgün, R. Voytovych, I. MacLaren, M. Rühle, R. M. Cannon, Cation segregation in an oxide ceramic with low solubility: Yttrium doped $\alpha$-alumina, Interface Science 10 (2002) 99-110.

[19] I. MacLaren, R. M. Cannon, M. A. Gülgün, R. Voytovych, N. Popescu-Pogrion, C. Scheu, U. Täffner, M. Rühle, Abnormal grain growth in alumina: Synergistic effects of yttria and silica, J. Am. Ceram. Soc. 86 (2003) 650-59.

[20] R. Brydson, P. C. Twigg, F. Loughran, F. L. Riley, Influence of CaO-SiO 2 ratio on the chemistry of intergranular films in liquid-phase sintered alumina and implications for rate of erosive wear, J. Mater. Res. 16 (2001) 652-682.

[21] D. L. Callahan, G. Thomas, Impurity distribution in polycrystalline alumina, J. Am. Ceram. Soc. 73 (1990) 2167-2170. 
[22] H. Wang, Y.-M. Chiang, Thermodynamic stability of intergranular amorphous films in bismuth-doped zinc oxide, Journal of the American Ceramic Society 81 (1998) 89-95.

[23] J. Luo, H. Wang, Y.-M. Chiang, Origin of solid-state activated sintering in $\mathrm{Bi}_{2} \mathrm{O}_{3}$-doped $\mathrm{ZnO}$, Journal of the American Ceramic Society 82 (1999) 916920.

[24] J. J. Cao, W. J. MoberlyChan, L. C. DeJonghe, C. J. Gilbert, R. O. Ritchie, In situ toughened silicon carbide with Al-B-C additions, Journal American Ceramic Society 79 (1996) 461-469.

[25] D. Chen, X.-F. Zhang, R. O. Ritchie, Effects of grain-boundary stucture on strength, toughness, and cyclic-fatigue properties of a monolithic silicon carbide, Journal American Ceramic Society 83 (2000) 2079-2081.

[26] Y.-M. Chiang, L. A. Silverman, R. H. French, R. M. Cannon, Thin glass film between ultrafine conductor particles in thick-film resistors, Journal of the American Ceramic Society 77 (1994) 1143-1152.

[27] Y.-M. Chiang, H. Wang, J.-R. Lee, HREM and STEM of intergranular films at zinc oxide varistor grain boundaries, J. Microscp. 191 (1998) 275-85.

[28] D. R. Clarke, Boundaries in electronic and superconducting ceramics, Col. de Physique 51 (1990) C1935-943.

[29] D. R. Clarke, On the equilibrium thickness of intergranular glass phases in ceramic materials, Journal of the American Ceramic Society 70 (1987) 15-22.

[30] D. R. Clarke, T. M. Shaw, A. P. Philipse, R. G. Horn, Possible electrical doublelayer contribution to the equilibrium thickness of intergranular glass films in polycrystalline ceramics, Journal of the American Ceramic Society 76 (1993) $1201-1204$. 
[31] D. R. Clarke, The intergranular film in silicon nitride ceramics: A diffuse interface approach, in: M. J. Hoffmann, G. Petzow (Eds.), Tailoring of Mechanical Properties of $\mathrm{Si}_{3} \mathrm{~N}_{4}$ Ceramics, Kluwer Academic Publishers, Amsterdam, 1994, pp. 291-301.

[32] H. D. Ackler, Y.-M. Chiang, Model experiment on thermodynamic stability of retained intergranular amorphous films, Journal of the American Ceramic Society 80 (1997) 1893-1896.

[33] M. Bobeth, D. R. Clarke, W. Pompe, A diffuse interface description of intergranular films in polycrystalline ceramics, Journal of the American Ceramic Society 82 (1999) 1537-1546.

[34] H.-J. Choi, G.-H. Kim, J.-G. Lee, Refined continuum model on the behavior of intergranular films in silicon nitride ceramics, Journal American Ceramic Society 83 (2000) 2821-2827.

[35] D. A. Litton, S. H. Garofalini, Molecular dynamics simulations of calcium aluminosilicate intergranular films on (0001) $\mathrm{Al}_{2} \mathrm{O}_{3}$ facets, Journal of the American Ceramic Society 83 (2000) 2273-2281.

[36] M. Yoshiya, I. Tanaka, H. Adachi, Energetical role of modeled intergranular glassy film in $\mathrm{Si}_{3} \mathrm{~N}_{4}-\mathrm{SiO}_{2}$ ceramics, Acta Materialia 48 (2000) 4641-4645.

[37] S. H. Garofalini, W. Luo, Molecular dynamics simulations of calcium silicate intergranular films between silicon nitride crystals, Journal of the American Ceramic Society 86 (2003) 1741-1752.

[38] X. Su, S. H. Garofalini, Atomistic structure of calcium silicate intergranular films between prism and basal planes in silicon nitride: A molecular dynamics study, J. Mater. Res. 19 (2004) 752-758.

[39] C. M. Bishop, R. E. García, W. C. Carter, Effect of charge separation on the stability of large wavelength fluctuations during spinodal decomposition, Acta 
Materialia 51 (2003) 1517-1524.

[40] R. E. García, C. M. Bishop, W. C. Carter, Thermodynamically consistent variational principles with applications to electrically and magnetically active systems, Acta Materialia 52 (2004) 11-21.

[41] R. Kobayashi, J. A. Warren, W. C. Carter, A continuum model of grain boundaries, Physica D 140 (2000) 141-150.

[42] C. M. Bishop, W. C. Carter, Relating atomistic grain boundary simulation results to the phase-field model, Computational Materials Science 25 (2002) $378-386$.

[43] C. M. Bishop, Continuum models for intergranular films in silicon nitride and comparison to atomistic simulations, Ph.D. thesis, Department of Materials Science and Engineering, Massachusetts Institute of Technology (2003).

[44] C. L. Johnson, M. J. Hÿtch, P. R. Buseck, Nanoscale waviness of low-angle grain boundaries, PNAS 101 (2004) 17936-17939.

[45] C. Koch, unpublished research.

[46] M. F. Yan, R. M. Cannon, H. K. Bowen, Space charge, elastic field, and dipole contributions to equilibrium solute segregation at interfaces, Journal of Applied Physics 54 (1983) 764-778.

[47] K. Johnston, M. W. Finnis, Electrostatic and entropic interactions between parallel interfaces separated by a glassy film, Journal of the American Ceramic Society 85 (2002) 2562-2568.

[48] Y.-M. Chiang, D. P. Birnie, W. D. Kingery, Physical Ceramics: Principles for Ceramic Science and Engineering, John Wiley and Sons, New York, 1997.

[49] U. Gerling, M. J. Pool, B. Predel, A contribution to the associate model for binary liquid alloys, Zeitschrift für Metallkunde 74 (1983) 616-619. 
[50] M. Hillert, The compound energy formalism, Journal of Alloys and Compounds 320 (2001) 161-176.

[51] A. A. Wheeler, W. J. Boettinger, G. B. McFadden, Phase-field model for isothermal phase transitions in binary alloys, Physical Review A 45 (1992) $7424-7439$.

[52] R. S. Qin, E. R. Wallach, A phase-field model coupled with a thermodynamic database, Acta Materialia 51 (2002) 6199-6210.

[53] L. Dumitrescu, B. Sundman, A thermodynamic reassessment of Si-Al-O-N system, Journal of the European Ceramic Society 15 (1995) 239-247.

[54] M. Hillert, S. Jonsson, B. Sundman, Thermodynamic calculation of the Si-N-O system, Zeitschrift für Metallkunde 83 (1992) 648-654.

[55] H. Gu, R. M. Cannon, H. J. Seifert, M. J. Hoffmann, I. Tanaka, Solubility of $\mathrm{Si}_{3} \mathrm{~N}_{4}$ in liquid $\mathrm{SiO}_{2}$, J. Am. Ceram. Soc. 85 (2002) 25-32.

[56] W. H. Press, S. A. Teukolsky, W. T. Vetterling, B. P. Flannery, Numerical Recipes in C, Cambridge University Press, Cambridge, 1992.

[57] J. W. Cahn, J. E. Hilliard, Free energy of a non-uniform system. I. Interfacial free energy, The Journal of Chemical Physics 28 (1958) 258-267.

[58] C. M. Bishop, M. Tang, R. M. Cannon, W. C. Carter, Continuum modelling of interfaces and their transitions in materials, submitted to Materials Scinece and Engineering A (2005).

[59] J. W. Cahn, Critical point wetting, Journal of Chemical Physics 66 (1977) $3667-3672$.

[60] M. Tang, W. C. Carter, Ordering transitions at grain boundaries in twocomponent systems, in preparation (2005). 


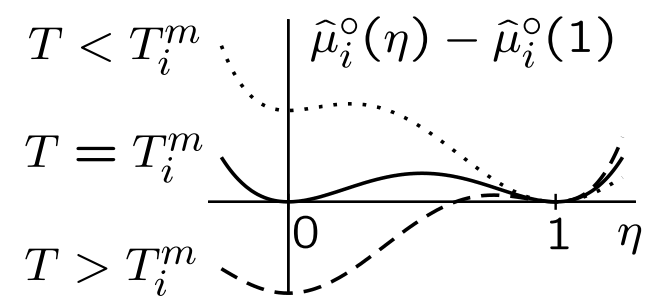

Fig. 1. Schematic of the normalized reference chemical potential, $\hat{\mu}_{i}^{\circ}(\eta)$, dependence on structure, $\eta$. The crystalline solid $(\eta=1)$ has the lowest free energy when $T<T_{i}^{m}$, the liquid $(\eta=0)$ has the lowest free energy when $T>T_{i}^{m}$.

[61] M. Tang, W. C. Carter, R. M. Cannon, Order-disorder structural transitions at grain boundaries in the KWC phase-field model, in preparation (2005).

[62] J. E. Taylor, Mean curvature and weighted mean curvature, overview 98(ii), Acta Materialia 40 (1992) 1475-1485.

[63] B. Nestler, A. A. Wheeler, Anisotropic multi-phase-field model: Interfaces and junctions, Physical Review E 57 (1998) 2602-2609.

[64] C. T. Koch, S. Bhattacharyya, A. Subramaniam, M. Rühle, Assessing thermodynamic properties of amorphous nanostructures by energy filtered electron diffraction, Microsc. Microanal. 10 (2004) 254-255. 


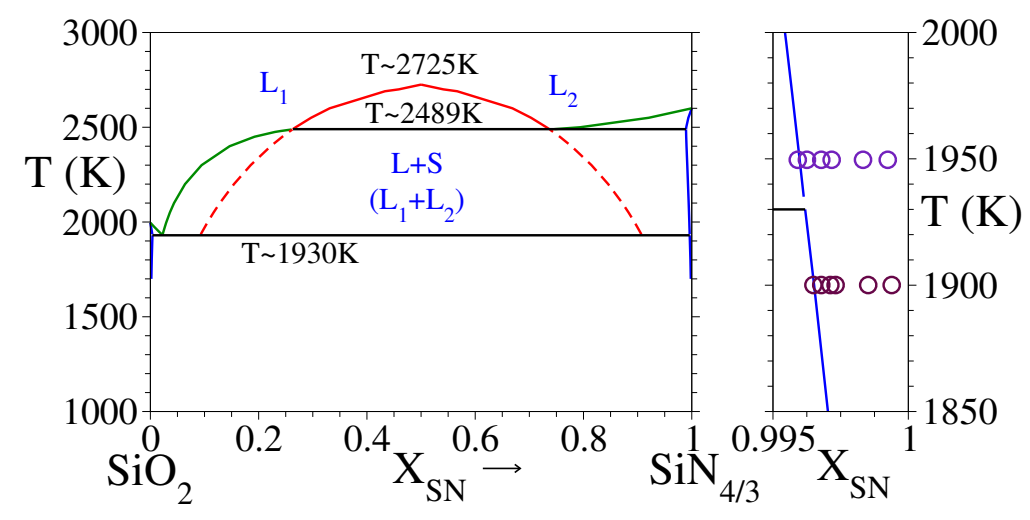

Fig. 2. Pseudo-binary phase diagram of the $\mathrm{SiO}_{2}-\mathrm{Si}_{3} \mathrm{~N}_{4}$ system calculated from bulk-equilibrium free energy densities and the approximations described in Section 2.3. The $\mathrm{Si}_{3} \mathrm{~N}_{4}$-rich side and the points corresponding to the bulk-equilibrium compositions used in the simulations are magnified to the right.

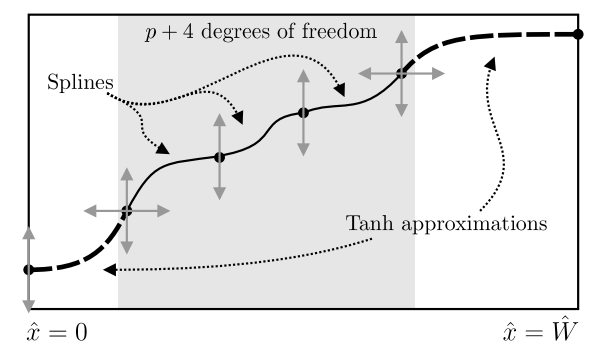

Fig. 3. Schematic of the parameterization of a field using a combination of tanh and cubic splines in the half space. There are $p+4$ points at which the function is specified and $p+7$ total parameters. The $p+2$ points between and including the end points of the tanh functions are interpolated with cubic splines. The arrows indicate the degrees of freedom at each point. The first derivatives are continuous in this scheme. 
(a)
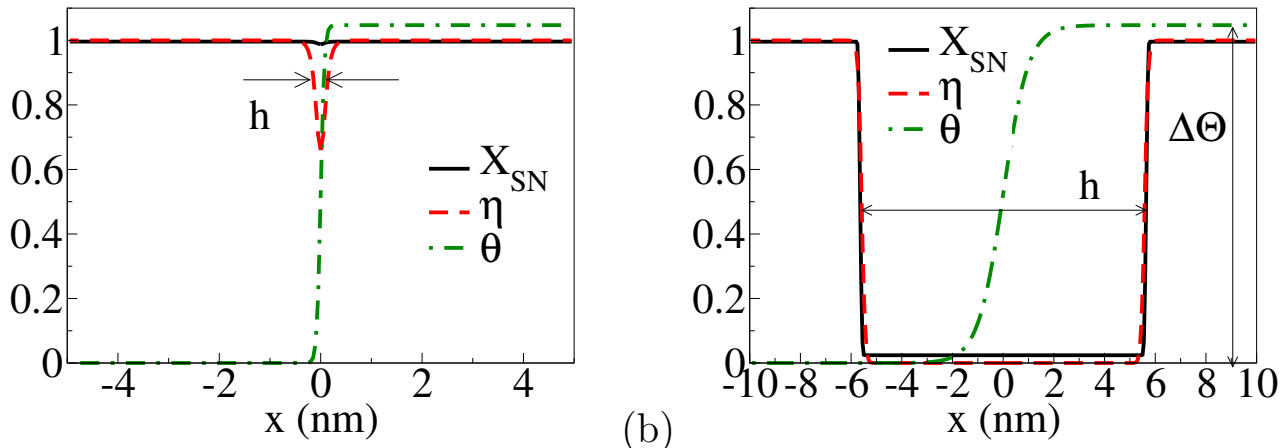

Fig. 4. Illustrations of stable solutions from simulated annealing with (a) ordered and (b) disordered boundaries.

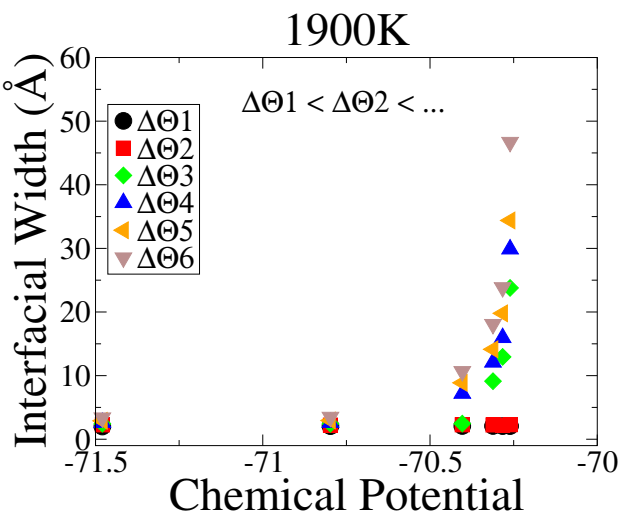

(b)

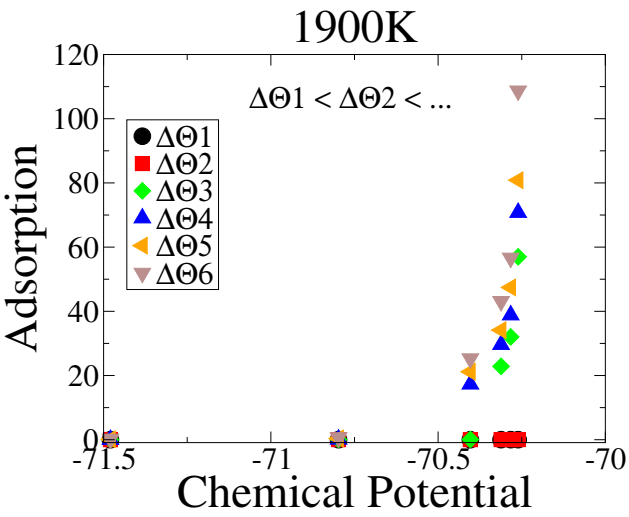

(c)

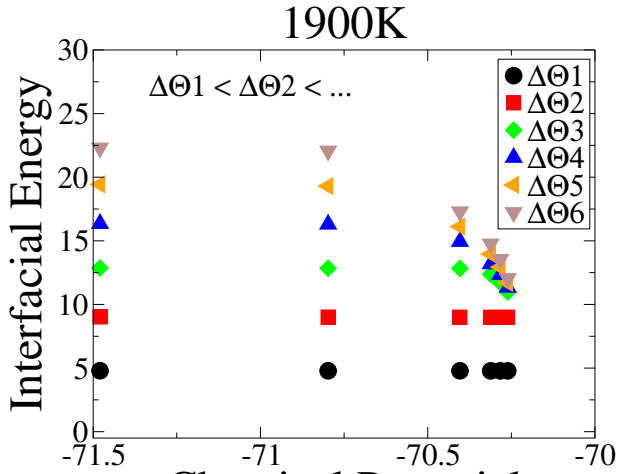

Chemical Potential

Fig. 5. Plots of the (a) interface width, (b) adsorption of $\mathrm{SiO}_{2}$, and (c) interfacial energy as a function of chemical potential of $\mathrm{SiO}_{2}$ at $1900 \mathrm{~K}$. The normalized gradient energy parameters are $\hat{\kappa}^{2}=10, \hat{s}=20, \hat{\beta}^{2}=10, \hat{\nu}^{2}=20$. 


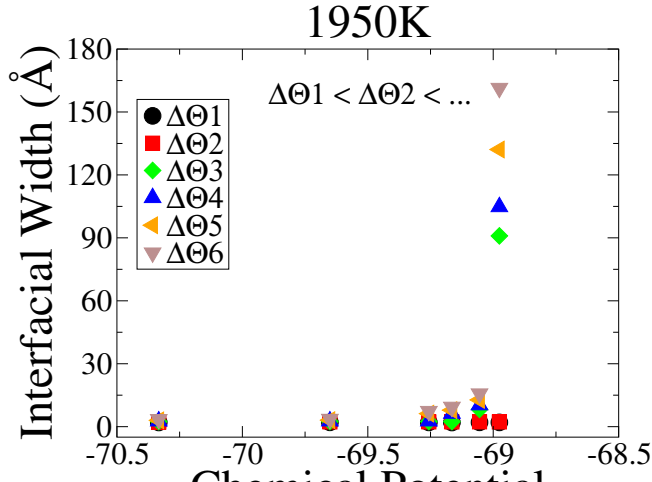

Chemical Potential

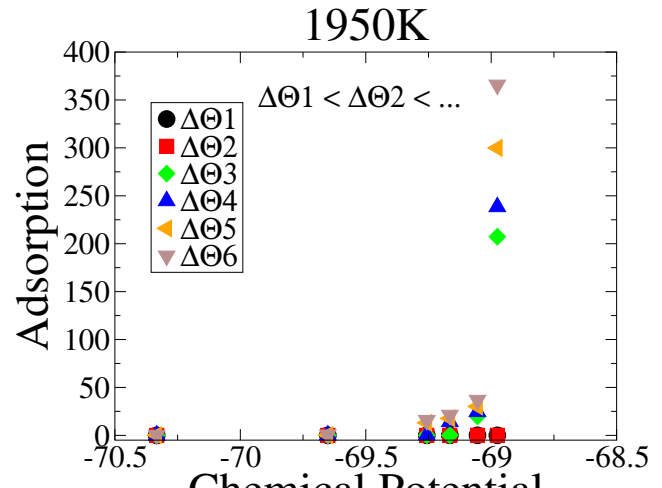

(b)

Chemical Potential

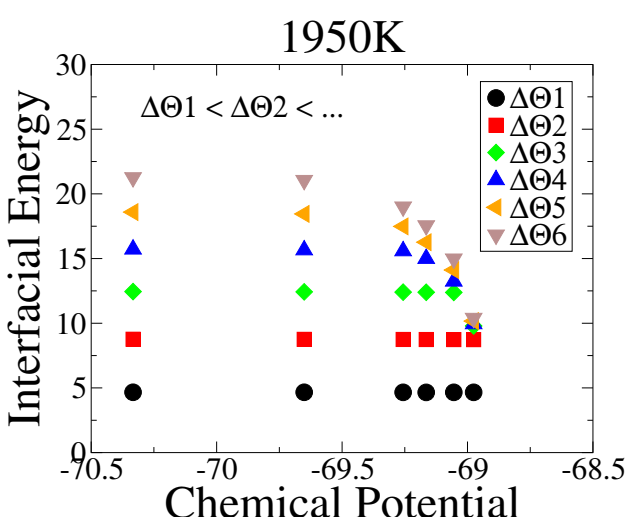

(c)

Chemical Potential

Fig. 6. Plots of the (a) interface width, (b) adsorption of $\mathrm{SiO}_{2}$, and (c) interfacial energy as a function of chemical potential of $\mathrm{SiO}_{2}$ at $1950 \mathrm{~K}$. The normalized gradient energy parameters are $\hat{\kappa}^{2}=9.744, \hat{s}=19.487, \hat{\beta}^{2}=9.744, \hat{\nu}^{2}=19.487$. 
(a)

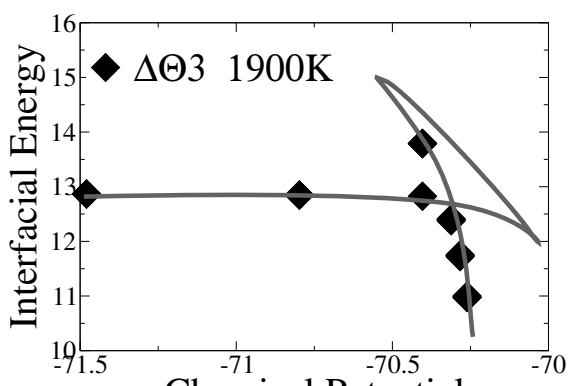

Chemical Potential

(b)

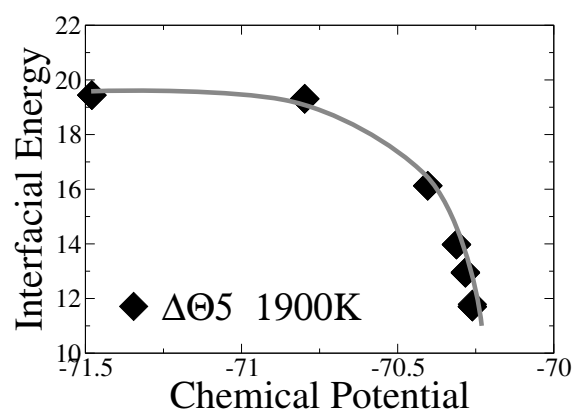

Fig. 7. Plots of the interfacial energy as a function of chemical potential for all simulated annealing results at (a) an "intermediate" and (b) a "large" misorientation. The grey curves are speculation on the disappearance of spinodal-like behavior for transitions between ordered and disordered interfaces. The data in (b) do not suffice to eliminate a metastable range as the simulated annealing technique may not find metastable solutions when a more stable solution is nearby. 